\title{
artigo
}

Santos, A.C.; Torres, V.S.F.; César, E.S.R.; Ferreira, J.D.L.; Oliveira, R.C.; Morais, C.A.C.;

Fatores que influenciam a segurança do paciente em hemodiálise: revisão integrativa

\section{Fatores que influenciam a segurança do paciente em hemodiálise: revisão integrativa}

\author{
Factors that influence patient safety in hemodialysis: integrative review
}

Factores que influyen en la seguridad del paciente en hemodiálisis: revisión integrativa

\begin{abstract}
RESUMO
Objetivo: Analisar as evidências científicas sobre a segurança do paciente submetido a hemodiálise. Método: Trata-se de uma revisão integrativa realizada nas bases de dados MEDLINE, LILACS, BDENF e IBECS, por meio da BVS, e SciELO no período de agosto a setembro de 2020. Um total de 14 artigos foi selecionado para integrar esta revisão. Resultados: A partir da análise das publicações, emergiram duas categorias temáticas: A enfermagem no cuidado ao paciente em terapia hemodialítica e Eventos adversos em unidades de hemodiálise: fatores que interferem na segurança do paciente. Os estudos destacam a infecção, hipotensão, dispneia e erros na administração de medicamentos, eventos frequentemente encontrados em unidades de hemodiálise. Conclusão: A análise das produções permitiu a compreensão dos principais fatores que interferem na segurança do paciente em tratamento hemodialítico, assim como a importância dos cuidados de enfermagem na implementação de ações voltadas à prevenção e ao tratamento de complicações.
\end{abstract}

DESCRITORES: Segurança do paciente; Diálise renal; Cuidados de enfermagem; Eventos Adversos.

\section{ABSTRACT}

Objective: To analyze the scientific evidence on the safety of patients undergoing hemo-dialysis. Method: This is an integrative review carried out in the MEDLINE, LILACS, BDENF and IBECS databases, through the VHL, and SciELO in the period from August to September 2020. A total of 14 articles were selected for this review. Results: From the analysis of the publications, two thematic categories emerged: Nursing in patient care in hemodialysis therapy and Adverse events in hemodialysis units: factors that interfere in the patient's safety. Studies highlight infection, hypotension, dyspnea and errors in medi-cation administration, events frequently found in hemodialysis units. Conclusion: The analysis of the productions allowed the understanding of the main factors that interfere in the safety of the patient in hemodialysis treatment, as well as the importance of nursing care in the implementation of actions aimed at the prevention and treatment of complica-tions.

DESCRIPTORS: Patient safety; Renal dialysis; Nursing care; Adverse events.

\section{RESUMEN}

Objetivo: Analizar la evidencia científica sobre la seguridad de los pacientes en hemodiá-lisis. Método: Se trata de una revisión integradora realizada en las bases de datos MEDLINE, LILACS, BDENF e IBECS, a través de la BVS, y SciELO en el período de agosto a septiembre de 2020. Para esta revisión se seleccionaron un total de 14 artículos. Resultados: del análisis de las publicaciones surgieron dos categorías temáticas: Enfer-mería en la atención al paciente en terapia de hemodiálisis y Eventos adversos en uni-dades de hemodiálisis: factores que interfieren en la seguridad del paciente. Los estudios destacan infección, hipotensión, disnea y errores en la administración de medicamentos, eventos que se encuentran con frecuencia en las unidades de hemodiálisis. Conclusión: El análisis de las producciones permitió comprender los principales factores que interfie-ren en la seguridad del paciente en el tratamiento de hemodiálisis, así como la importan-cia del cuidado de enfermería en la implementación de acciones orientadas a la preven-ción y tratamiento de complicaciones.

DESCRIPTORES: Seguridad del paciente; Diálisis renal; Cuidado de enfermera; Eventos adversos.

RECEBIDO EM: 26/01/2021 APROVADO EM: 10/02/2021

\section{Anna Cecília dos Santos}

Enfermeira graduada pela Faculdade de Enfermagem Nova Esperança.

ORCID: 0000-0002-2508-5384 


\section{Valdicléia da Silva Ferreira Torres}

Enfermeira. Mestre em Enfermagem pelo Programa de Pós-graduação em Enfermagem da UFPB.

ORCID: 0000-0002-3974-7123

\section{Edna Samara Ribeiro César}

Enfermeira. Mestre em Ciências da Nutrição pela Universidade Federal da Paraíba e Mestre em terapia Intensiva pelo Instituto Brasileiro de Terapia Intensiva. Docente do curso de graduação em Enfermagem da Faculdade de Enfermagem Nova Esperança (FACENE). ORCID: 0000-0001-7642-2558

\section{Josefa Danielma Lopes Ferreira}

Enfermeira. Doutoranda em Enfermagem pelo Programa de Pós-graduação em Enfermagem da UFPB.

ORCID: 0000-0003-4209-4781

\section{Regina Célia de Oliveira}

Enfermeira. Pós-Doutorado pela Escola de Enfermagem de Ribeirão Preto.

ORCID: 0000-0002-6559-5872

\section{Camila Abrantes Cordeiro Morais}

Enfermeira. Doutoranda em Enfermagem pelo Programa Associado de Pós-graduação em Enfermagem da UPE-UEPB. ORCID: 0000-0003-3780-9340

\section{INTRODUÇÃO}

$\mathbf{N}$ os últimos anos, têm-se elevado as discussões acerca da Doença Renal Crônica (DRC), decorrente do aumento de sua prevalência entre a população mundial e por seu significativo impacto na morbimortalidade dos indivíduos acometidos, tornando-se um problema de saúde pública. ${ }^{1}$

A DRC é definida como uma lesão do parênquima renal, que, por sua vez, acarreta anormalidades estruturais e funcionais que comprometem a funcionalidade dos rins por um período igual ou superior a três meses ${ }^{2}$. No Brasil, os dados revelam que cerca de 12 milhões de pessoas apresentam algum grau de insuficiência renal e que a incidência de IRC aumenta em torno de $8 \%$ ao ano. ${ }^{3,4}$

No estágio avançado da doença, o indivíduo necessita de um complexo tratamento que envolve a necessidade de Terapias de Substituição Renal (TRS), associado a restrições alimentar e hídrica, além de administração de medicamentos ${ }^{5,6}$. A hemodiálise, uma das principais modalidades de TRS, submete o paciente à necessidade de um dispositivo extracorpóreo de filtração sanguínea que, por meio de uma membrana semipermeável, promove as trocas de líquidos e eletrólitos. ${ }^{7,8}$

É importante destacar que a hemodiálise envolve mecanismos complexos que, muitas vezes, favorecem o desenvolvimento de eventos adversos (EAs), que são definidos como incidentes que ocorrem durante a assistência à saúde e que resultam em dano ao paciente, o que inclui doença, lesão, sofrimento, incapacidade ou morte. ${ }^{9-11}$

A supervisão do paciente submetido a hemodiálise é primordial para assegurar uma assistência segura, sendo imprescindível que os profissionais sejam capacitados para identificação e intervenção diante de possíveis complicações no tratamento. A participação do enfermeiro no tratamento é imprescindível, viabilizando apoio e informando ao paciente, bem como à sua família, sobre sua patologia e as dificuldades que podem ser encontradas. ${ }^{12}$

Nessa perspectiva, tornam-se relevantes estudos acerca da segurança do paciente nos serviços hospitalares, especialmente em unidades de hemodiálise, tendo em vista que representam locais susceptíveis a ocorrência de EAs, a partir da interação de diversos fatores. Tal pesquisa contribui para a ampliação do conhecimento sobre a temática na área da enfermagem, auxiliando no planejamento de ações de identificação, prevenção e controle dos EAs associados ao tratamento com hemodiálise. Sendo assim, este estudo tem como objetivo analisar as publicações científicas sobre a segurança do paciente submetido a hemodiálise.

\section{MÉTODO}

Trata-se de uma Revisão Integrativa da Literatura, em que foram percorridas as seguintes etapas para a realização deste estudo: identificação de problema, com a definição da questão de pesquisa; estabelecimento de critérios para inclusão e/ou exclusão de estudos para a busca de literatura científica; definição das informações a serem extraídas dos estudos; avaliação dos estudos; interpretação dos resultados e apresentação da revisão/ síntese do conhecimento13.Atendendo ao objetivo anteriormente descrito, foi definida a seguinte questão de pesquisa:"Quais as evidências científicas sobre a segurança do paciente submetido a hemodiálise?".

A busca foi realizada em quatro bases de dados: Medical Literature Analysis and Retrieval System Online (MEDLINE), Literatura Latino-Americana do Caribe em Ciências da Saúde (LILACS), Bases de Dados de Enfermagem (BDENF) e Índice Bibliográfico Espanhol de Ciências da Saúde (IBECS), por meio do Portal da Biblioteca Virtual em Saúde (BVS). Para melhor selecionar os arquivos, também se recorreu ao banco de periódicos Scientific Electronic Library Online (SciELO).

Foram utilizados os termos identificados no vocabulário na base dos Descritores em Ciências da Saúde (DeCS) e no Medical Subject Headings (MeSH). Assim, foi 


\section{artigo}

Santos, A.C.; Torres, V.S.F.; César, E.S.R.; Ferreira, J.D.L.; Oliveira, R.C.; Morais, C.A.C.

Fatores que influenciam a segurança do paciente em hemodiálise: revisão integrativa.

aplicada uma combinação dos descritores "Segurança do paciente", "Patient Safety", "Unidades Hospitalares de Hemodiálise", "Hemodialysis Units Hospital”, "Cuidados de enfermagem" e "Nursing care", separados pelo operador booleano AND.

A coleta de dados ocorreu no período de agosto a setembro de 2020. Para selecionar a amostra, foram adotados os seguintes critérios de elegibilidade: publicações na modalidade artigo, texto com- pleto, publicados no período de 2010 a 2020, nos idiomas português, espanhol e inglês. Foram excluídas publicações como: teses, dissertações, monografias, trabalhos de conclusão de curso, relatos de caso, relatos de experiência, revisão de literatura, manuais, resenhas, notas prévias e artigos que não abordavam a temática proposta.

Para a seleção dos estudos, seguimos as recomendações do método Preferred Reporting Items for Systematic reviews and

Figura 1: Fluxograma do processo de seleção dos artigos incluídos na revisão integrativa atendendo aos critérios PRISMA14, João Pessoa, Paraỉba, Brasil, 2020.

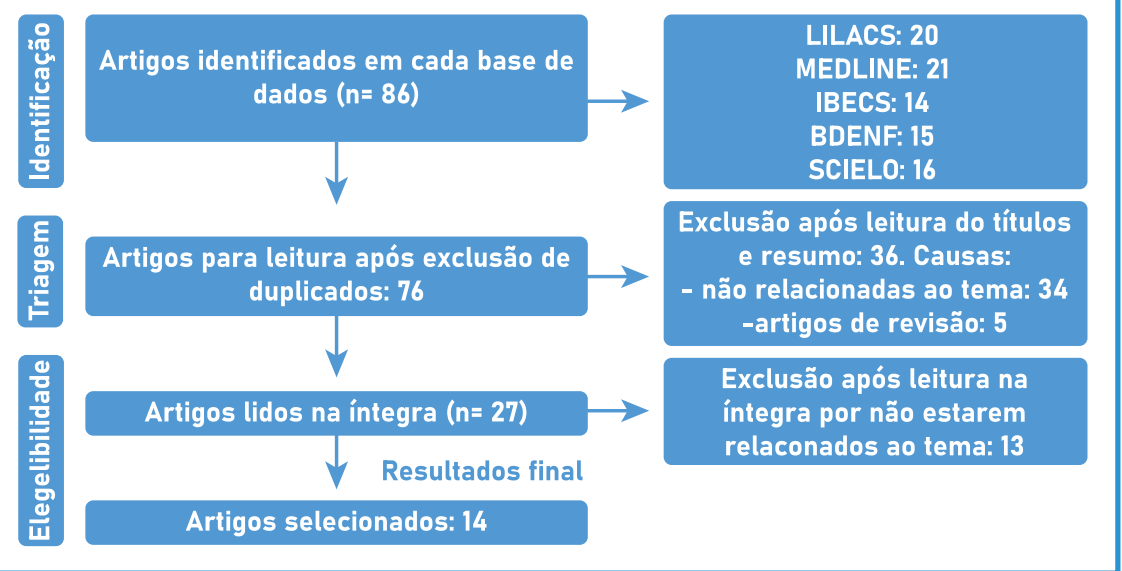

Fonte: Fluxograma elaborado pelos autores, 2020

Meta-Analyses - PRISMA14 (Figura 1). Assim, os artigos foram inicialmente selecionados pelo título e resumo e, posteriormente, lidos na íntegra, incluindo-se os que continham informações relevantes acerca da segurança do paciente em tratamento hemodialítico. Inicialmente, foi identificado um total de 86 publicações. Após as etapas de identificação, triagem e elegibilidade, a amostra foi composta por 14 artigos.

Para a coleta e análise dos dados, utilizou-se de um formulário padronizado que abordou as seguintes variáveis: título do artigo, autores, ano de publicação, delineamento e objetivo do estudo.

\section{RESULTADOS}

Dos 14 artigos que compuseram a amostra final, $10(71,4 \%)$ foram publicados em periódicos internacionais e 4 (28,5\%) em periódicos nacionais. Referente ao ano de publicação, identifica-se que houve maior produção nos anos de 2017 (28,5\%) e 2019 $(21,4 \%)$. Ressalta-se que não foi localizada nenhum artigo publicado nos anos de 2010 e 2012, conforme observado no Quadro 1.

Verifica-se, no Quadro 2, que em relação delineamento metodológico, a maioria das

\section{Quadro 1: Distribuição dos artigos selecionados para a revisão integrativa. João Pessoa, Paraíba, Brasil, 2020.}

\begin{tabular}{|c|c|}
\hline ANO & AUTOR/TÍTULO/PERIÓDICO \\
\hline 2019 & $\begin{array}{l}\text { Cuevas-Budhart, MA et al. Diseño y validación de un nuevo registro clínico de enfermería, para la continuidad de los cuidados y } \\
\text { seguridad del paciente en hemodiálisis. Revista Enfermería Nefrológica. }\end{array}$ \\
\hline 2019 & $\begin{array}{l}\text { López VEG, Macías CM, Cuestas RC, de Lara MAA, Montero MC. Análisis de las medidas correctoras para la disminución de los } \\
\text { eventos adversos en una unidad de hemodiálisis hospi-talaria. Revista de la Sociedad Española de Enfermería Nefrológica. }\end{array}$ \\
\hline 2019 & $\begin{array}{c}\text { Cuevas-Budhart, MA et al. Factores asociados al desarrollo de eventos adversos en pacientes con hemodiálisis en Guerrero, } \\
\text { México. Revista Enfermería Nefrológica. }\end{array}$ \\
\hline 2018 & $\begin{array}{l}\text { Pássaro, PG. D'Ávila, R. Nursing educational intervention for the identification of Adverse Events in hemodialysis. Revista Brasi- } \\
\text { leira de Enfermagem. }\end{array}$ \\
\hline 2018 & $\begin{array}{l}\text { Lessa SRO, Bezerra JNM, Barbosa SMC, Luz GOA, Borba AKOT. Prevalência e fatores associados para a ocorrência de eventos } \\
\text { adversos no serviço de hemodiálise. Revista Texto \& Contexto Enfermagem. }\end{array}$ \\
\hline 2017 & $\begin{array}{l}\text { Jimenéz MDA, Ferre G, Álvarez-Ude F. Estrategias para aumentar la seguridad del paciente en hemodiálisis: Aplicación del } \\
\text { sistema de análisis modal de fallos y efectos (sistema AMFE). Revista Enfermería Nefrológica. }\end{array}$ \\
\hline 2017 & $\begin{array}{l}\text { Barrios S, Catoni MI, Arechabala MC, Palma E, Ibacache Y, Richard J. Carga laboral de las enfermeras en Unidades de Hemodiáli- } \\
\text { sis Crónica según dependencia y riesgo de los pacientes. Revista médica de Chile. }\end{array}$ \\
\hline 2017 & $\begin{array}{c}\text { Aguiar LL, Guedes MVC, Oliveira RM, Leitão IMTA, Pennafort VPS, Barros AA.Nursing and international safety goals: hemodialy- } \\
\text { sis assessment. Cogitare Enfermagem. }\end{array}$ \\
\hline 2017 & $\begin{array}{l}\text { Lima-Aguiar, L. Cavalcante-Guedes, MV. Diagnósticos e intervenciones de enfermería del dominio seguridad y protección de los } \\
\text { pacientes en hemodiálisis. Enfermería global. }\end{array}$ \\
\hline
\end{tabular}




\begin{tabular}{|c|c|}
\hline 2016 & $\begin{array}{c}\text { Guerrero VM, García GP, Hidalgo CGO, Henández de Arribas V, Penã JR. Nivel de seguri-dad percibida por el paciente de hemo- } \\
\text { diálisis crónica. Revista Enfermería Nefrológica. }\end{array}$ \\
\hline 2015 & $\begin{array}{c}\text { Prezerakos, P. Galanis, P. Moisoglou, I. The work environment of haemodialysis nurses and its impact on patients' outcomes. } \\
\text { International Journal of Nursing Practice. }\end{array}$ \\
\hline 2014 & $\mathrm{Gu}$, X. Itoh, K. Suzuki, S. An error taxonomy system for analysis of haemodialysis incidents. Journal of Renal Care. \\
\hline 2013 & $\begin{array}{c}\text { Sousa MRG, Silva AEBC, Bezerra ALQ. Eventos adversos em hemodiálise: relatos de profissionais de enfermagem. Revista da } \\
\text { Escola de Enfermagem da USP. }\end{array}$ \\
\hline 2011 & $\begin{array}{c}\text { Quori A, Baamonde-Laborda E, García-Cantón C, Lago-Alonso MM, Toledo-Gonzáles A, Monzón-Jiménez E, et al. Vigilancia de } \\
\text { infecciones y otros eventos adversos en pacientes en diálisis en el área sur de Gran Canaria. Revista Enfermería Nefrológica. }\end{array}$ \\
\hline Fonte: dados da pesquisa. \\
\hline
\end{tabular}

publicações analisadas foram originadas de estudos transversais $(28,5 \%)$, sendo a abordagem quantitativa a mais prevalente $(78,5 \%)$.

\section{DISCUSSÃO}

A partir da análise dos estudos seleciona- dos, emergiram duas categorias temáticas: A enfermagem no cuidado ao paciente em terapia hemodialítica; e Eventos adversos em

\begin{tabular}{|c|c|c|}
\hline Cód. & TIPO DE ESTUDO E ABORDAGEM & OBJETIVO \\
\hline E1 & $\begin{array}{l}\text { Estudo metodológico/ abordagem } \\
\text { mista }\end{array}$ & $\begin{array}{l}\text { Conceber e validar um instrumento para a melhoria do processo assistencial de enfer- } \\
\text { magem em uma unidade de hemodiálise de um hospital de nível médio. }\end{array}$ \\
\hline E2 & $\begin{array}{l}\text { Estudo retrospectivo de } \\
\text { abordagem quantitativa }\end{array}$ & $\begin{array}{c}\text { Avaliar a eficácia das medidas corretivas em um plano de segurança do paciente em } \\
\text { hemodiálise para reduzir eventos adversos. }\end{array}$ \\
\hline E3 & $\begin{array}{l}\text { Estudo retrospectivo de aborda- } \\
\text { gem quantitativa }\end{array}$ & $\begin{array}{l}\text { Determinar os eventos adversos mais prevalentes e os fatores associados ao seu de- } \\
\text { senvolvimento no paciente em hemodiálise em Guerrero, México. }\end{array}$ \\
\hline E4 & $\begin{array}{l}\text { Estudo quase experimental, pros- } \\
\text { pectivo, de abordagem quantitativa }\end{array}$ & $\begin{array}{l}\text { Construir um programa educacional voltado para a capacitação dos técnicos de Enfer- } \\
\text { magem, que possibilite a compreensão dos Eventos Adversos. }\end{array}$ \\
\hline E5 & $\begin{array}{l}\text { Estudo descritivo longitudinal com } \\
\text { abordagem quantitativa }\end{array}$ & $\begin{array}{l}\text { Analisar a prevalência e os fatores associados para a ocorrência de eventos adversos no } \\
\text { serviço de hemodiálise. }\end{array}$ \\
\hline E6 & $\begin{array}{l}\text { Estudo retrospectivo de aborda- } \\
\text { gem quantitativa }\end{array}$ & $\begin{array}{l}\text { Mostrar um sistema de trabalho, reproduzivel em qualquer unidade de hemodiálise, que } \\
\text { consiste em registrar as complicações e falhas ocorridas durante a sessão. }\end{array}$ \\
\hline E7 & $\begin{array}{l}\text { Estudo transversal de abordagem } \\
\text { quantitativa }\end{array}$ & $\begin{array}{l}\text { Determinar a carga de trabalho do enfermeiro segundo perfis de dependência e risco dos } \\
\text { pacientes em hemodiálise crônica. }\end{array}$ \\
\hline E8 & $\begin{array}{l}\text { Estudo descritivo de abordagem } \\
\text { qualitativa }\end{array}$ & $\begin{array}{c}\text { Descrever o cuidado de enfermagem prestado em serviço hospitalar de hemodiálise com } \\
\text { base em metas internacionais de segurança do paciente. }\end{array}$ \\
\hline E9 & $\begin{array}{l}\text { Estudo descritivo de abordagem } \\
\text { quantitativa }\end{array}$ & $\begin{array}{l}\text { Identificar os diagnósticos de enfermagem do domínio segurança e proteção da Taxo- } \\
\text { nomia II da NANDA-I e propor intervenções e atividades de enfermagem com base na } \\
\text { Classificação das Intervenções de Enfermagem. }\end{array}$ \\
\hline E10 & $\begin{array}{l}\text { Estudo transversal de abordagem } \\
\text { mista }\end{array}$ & Avaliar o nível de segurança percebido pelo paciente durante a sessão de hemodiálise. \\
\hline E11 & $\begin{array}{l}\text { Estudo transversal de abordagem } \\
\text { quantitativa }\end{array}$ & $\begin{array}{c}\text { Avaliar o ambiente de trabalho dos enfermeiros em hemodiálise e investigar a correlação } \\
\text { entre ambiente de trabalho e resultados dos pacientes na Grécia. }\end{array}$ \\
\hline E12 & $\begin{array}{l}\text { Estudo descritivo com abordagem } \\
\text { quantitativa }\end{array}$ & $\begin{array}{l}\text { Descrever o desenvolvimento de um sistema de taxonomia de erros de hemodiálise para } \\
\text { analisar incidentes e prever o status de segurança de uma organização de diálise no Japão. }\end{array}$ \\
\hline E13 & $\begin{array}{l}\text { Estudo transversal de abordagem } \\
\text { quantitativa }\end{array}$ & $\begin{array}{l}\text { Analisar o conhecimento dos profissionais de enfermagem sobre Eventos Adversos em } \\
\text { uma unidade de hemodiálise de um hospital de ensino. }\end{array}$ \\
\hline E14 & $\begin{array}{l}\text { Estudo prospectivo de abordagem } \\
\text { quantitativa }\end{array}$ & $\begin{array}{c}\text { Analisar e conhecer as características epidemiológicas dos eventos adversos (infecciosas } \\
\text { e não infecciosas) e identificar oportunidades potenciais de melhoria. }\end{array}$ \\
\hline
\end{tabular}


unidades de hemodiálise: fatores que interferem na segurança do paciente.

\section{A enfermagem no cuidado ao pa- ciente em terapia hemodialítica}

Um estudo realizado no Chile evidenciou que, durante uma sessão de hemodiálise, que apresenta duração média de 4 horas, o enfermeiro passa $60 \%$ do tempo prestando assistência direta ao paciente, incluindo cuidados ao acesso vascular, suporte emocional e administração de medicamentos. Constatou-se a necessidade de distribuição dos pacientes durante o plantão, a fim de evitar sobrecarga e estresse para os profissionais, o que pode favorecer a ocorrência de eventos adversos. ${ }^{15,16}$

Ao avaliar o ambiente de trabalho nos setores de hemodiálise, pesquisa ${ }^{17}$ realizada na Grécia descreveu o enfermeiro como o membro da equipe mais elegível para julgar a eficácia e eficiência de programas de segurança ao paciente. Outros estudos ${ }^{18,19}$ enfatizam que a atuação do enfermeiro frente a pacientes com DRC em tratamento hemodialítico, deve incluir o incentivo ao autocuidado, a inclusão da família no processo saúde-doença, além de orientações quanto a prevenção de infecção.

Risco de infecção, risco de sangramento, risco de contaminação e risco de hipotermia foram os principais diagnósticos de enfermagem referentes ao domínio de Segurança e Proteção do NANDA-I, presentes em um serviço de hemodiálise em Fortaleza-CE. As principais intervenções de enfermagem elencadas incluíam o controle de infecção, precauções contra sangramento, terapia de hemodiálise, controle de anafilaxia e regulação de temperatura. ${ }^{20}$

\section{Eventos adversos em unidades de hemodiálise: fatores que interferem na segurança do paciente}

As unidades de diálise são organizações complexas, que envolvem equipes multi- disciplinares e utilizam tecnologia avançada para cuidar de doentes com patologias múltiplas e graves. $\mathrm{O}$ acontecimento de EAs em centros de hemodiálise é preocupante, visto que qualquer erro pode acarretar consequências graves à saúde do paciente, que já está vulnerável e em condições clínicas desfavoráveis..$^{21,22}$

Diversos estudos ${ }^{18,23,24}$ destacam infecção, hipotensão, dispneia, erros durante a administração da medicação e cefaleia como sendo os eventos mais encontrados em pacientes durante a hemodiálise. Tais complicações estão associadas ao alto risco de morbimortalidade, necessitando da equipe o contínuo monitoramento, a deteç̧ão e intervenção precoce frente as complicações.

$\mathrm{O}$ treinamento em serviço e a realização de atividades de educação continuada são essenciais para que se promova discussão acerca temática segurança do paciente e de melhores práticas na assistência à saúde. No contexto da hemodiálise, a implementação de estratégias direcionadas à criação de protocolos para os cuidados durante a anticoagulação do circuito extracorpóreo, ao incentivo do registro de EA durante a hemodiálise em prontuário específico, assim como atualizações referentes ao manejo dos acessos vasculares, obtiveram resultados positivos na redução da incidência de eventos adversos. ${ }^{9,25-28}$

Ao investigar os eventos adversos mais prevalentes em paciente submetidos a hemodiálise, pesquis ${ }^{28}$ realizada no México concluiu que a infecção relacionada ao acesso vascular é fator determinante do número de internações. Fatores como a obesidade, desnutrição, hipercalemia e diabetes foram destacados como preditores de mortalidade em pacientes renais que, ao realizar hemodiálise, podem apresentar maior grau de resposta inflamatória. ${ }^{29}$

No Japão, também foi realizado um estudo com a criação de um sistema denominado "Taxonomia de Erro" e observou-se que mais de $70 \%$ dos incidentes de hemodiálise foram relatados como problemas ou complicações relacionadas ao dialisador, circuito ou medicação, e que aproximadamente $70 \%$ dos erros ocorreram imediatamente antes e depois das quatro horas de terapia. ${ }^{30}$

Diante do exposto, é importante enfatizar a necessidade de investigações, notificações e análises das ocorrências que auxiliem no planejamento de intervenções para reduzir a incidência dos EA, tornando, assim, o cuidado seguro e com menores riscos ao paciente ${ }^{31}$. Medidas como notificações, implementação de protocolos e educação contínua devem ser adotadas para a prevenção de EA, adotando estratégias para melhoria dos processos de cuidado desenvolvidos na prática diária.

\section{CONCLUSÃO}

Diante do objetivo proposto, conclui-se que as unidades de hemodiálise apresentam um grande potencial de risco para a ocorrência de eventos adversos, sendo necessário o incentivo à cultura de segurança do paciente nesses setores. Neste cenário, ressalta-se a importância da sistematização da assistência de enfermagem, proporcionando o cuidado contínuo a estes indivíduos, com organização e planejamento de voltadas à prevenção e tratamento de complicações associadas ao procedimento.

Como limitações do estudo, evidencia-se o reduzido número de publicações selecionadas para a amostra pertencentes a bases de dados internacionais, além da utilização dos descritores, que pode ter excluído pesquisas sobre a temática. Desse modo, sugere-se o desenvolvimento de novas investigações que possam contribuir para a criação de protocolos institucionais que auxiliem na organização dos cuidados ao paciente em hemodiálise, bem como na prática da educação permanente dos profissionais que atuam na assistência. "

\section{REFERÊNCIAS}

1. Sesso RC, Lopes AA, Thomé FS, Lugon JR, Martins CT. Inquérito Brasileiro de Diálise Crônica 2014. J Bras Nefrol. 2016;38(1):54-61.
2. Mello MVFA, Menezes KSP, Pires KKC, Angelo M. Panorama da doença renal terminal em um estado da amazônia brasileira. 


\section{REFERÊNCIAS}

2017;21:e994.

3. Sesso RC, Lopes AA, Thomé FS, Lugon JR, Martins CT. Inquérito Brasileiro de Diálise Crônica 2016. J Bras Nefrol. 2017;39(3):261266.

4. Oliveira ACR, Vieira DSR, Bündchen DC. Nível de atividade física e capacidade funcional de pacientes com doença renal crônica pré-dialítica e em hemodiálise. Fisioter Pesqui. 2018;25(3):323329.

5. Dallacosta FM, Dallacosta H, Mistrus L. Detecção precoce de doença renal crônica em população de risco. Cogitare Enferm. 2017;22(2):e48714.

6. Lins SMSB, Leite JL, Godoy S, Fuly PSC, Araújo STC, Silva IR. Adaptação cultural do questionário de adesão do paciente renal crônico em hemodiálise. Rev Bras Enferm. 2017;70(6):1169-75.

7. Debone MC, Pedruncci ESN, Candido MCP, Marques S, Kusumota L. Diagnósticos de enfermagem em idosos com doença renal crônica em hemodiálise. Rev Bras Enferm, 2017;70(4):800-5.

8. Xavier SSM, Germano RM, Silva IP, Lucena SKP, Martins JM, Costa IKF. Na correnteza da vida: a descoberta da doença renal crônica. Interface. 2018: 22(66):841-51.

9. Lessa SRO, Bezerra JNM, Barbosa SMC, Luz GOA, Borba AKOT. Prevalência e fatores associados para a ocorrência de eventos adversos no serviço de hemodiálise. Texto Contexto Enferm. 2018;27(3):e3830017.

10. Xelegati R, Gabriel CS, Dessotte CAM, Zen YP, Évora YDM. Eventos adversos relacionados ao uso de equipamentos e materiais na assistência de enfermagem a pacientes hospitalizados. Rev Esc Enferm USP. 2019; 53:e03503.

11. Brasil. Agência Nacional de Vigilância Sanitária (ANVISA), Cirurgias seguras salvam vidas. Brasília (DF);2008.

12. Frazão CM, Medeiros AB, Silva FB, Sá JD, Lira AL. Diagnósticos de enfermagem em pacientes renais crônicos em hemodiálise. Acta Paul Enferm. 2014;27(1):40-3.

13. Souza MT, Silva MD, Carvalho R. Revisão integrativa: o que é e como fazer. Rev Ein-stein. 2010;8(1):102-6.

14. Moher D, Liberati A, Tetzlaff J, Altman DG; PRISMA Group. Preferred reporting items for systematic reviews and meta-analyses: the PRISMA statement. Int J Surg. 2010;8(5):336-41.

15. Barrios S, Catoni MI, Arechabala MC, Palma E, Ibacache $Y$, Richard J. Carga laboral de las enfermeras en Unidades de Hemodiálisis Crónica según dependencia y riesgo de los pacientes. Rev Med Chile. 2017;145:888-895.

16. Sousa MRG, Silva AEBC, Bezerra ALQ, Freitas JS, Miasso Al. Eventos adversos em hemodiálise: relatos de profissionais de enfermagem. Rev Esc Enferm USP. 2013;47(1):76-83.

17. Prezerakos P, Galanis P, Moisoglou I. The work environment of haemodialysis nurses and its impact on patients' outcomes. Int J Nurs Pract. 2014;40(4):239-48.

18. Lucena AF, Magro CZ, Proença MCC, Pires AUB, Moraes VM,
Aliti GB. Validação de intervenções e atividades de enfermagem para pacientes em terapia hemodialítica. Rev Gaúcha Enferm. 2017;38(3):e66789

19. Oliveira ACF, Vieira DSR, Bündchen DC. Nível de atividade física e capacidade funcional de pacientes com doença renal crônica pré-dialítica e em hemodiálise. Fisioter Pesqui. 2018;25(3):323329.

20. Lima-Aguiar L, Cavalcante-Guedes MV. Diagnósticos e intervenciones de enfermería del dominio seguridad y protección de los pacientes en hemodiálisis. Enferm glob. 2017;16(47):1-12.

21. Costa RHS, Dantas ALM, Leite EMD, Lira ALBC, Vitor AF, Silva RAR. Complicações em pacientes renais durante sessões hemodialíticas e intervenções de enfermagem. J res: fundam care online. 2015;7(1):2137-2146.

22. Sousa MRG, Silva AEBC, Bezerra ALQ. Prevalência de eventos adversos em uma unidade de hemodiálise. Rev enferm UERJ. 2016;24(6):e18237.

23. Aguiar LL, Guedes MVC, Oliveira RM, Leitão IMTA, Pennafort VPS, Barros AA. Enfermagem e metas internacionais de segurança: avaliação em hemodiálise. Cogitare enferm. 2017;22(3):01-09.

24. Quori A, Baamonde-Laborda E, García-Cantón C, Lago-Alonso MM, Toledo-Gonzáles A, Monzón-Jiménez E, et al. Vigilancia de infecciones y otros eventos adversos en pa-cientes en diálisis en el área sur de Gran Canaria. Nefrología (Madr.). 2011;31(4):457-463.

25. López VEG, Macías CM, Cuestas RC, de Lara MAA, Montero MC. Análisis de las me-didas correctoras para la disminución de los eventos adversos en una unidad de hemo-diálisis hospitalaria. Enferm nefrol. 2019;22(1):27-33.

26. Cuevas-Budhart MAC, Martínez BRG, Bolaños EA, García $M J B$, Souza $A A$, Jurado $M A G$, et al. Diseño y validación de un nuevo registro clínico de enfermería, para la conti-nuidad de los cuidados y seguridad del paciente en hemodiálisis. Enferm nefrol. 2019;22(2):168-175.

27. Jimenéz MDA, Ferre G, Álvarez-Ude F. Estrategias para aumentar la seguridad del paciente en hemodiálisis: Aplicación del sistema de análisis modal de fallos y efectos (sistema AMFE). Nefrología (Madr.). 2017;37(6):608-621.

28. Cuevas-Budhart MAC, García RPS, Larumbe JAG, Bolaños EA, del Cerro EP, Monroy AM, et al. Factores asociados al desarrollo de eventos adversos en pacientes con hemo-diálisis en Guerrero, México. Enferm nefrol. 2019;22(1):42-50.

29. Pássaro PG, D'Ávila R. Nursing educational intervention for the identification of Ad-verse Events in hemodialysis. Rev bras enferm. 2018;71(4):1507-1513.

30. Gu X, Itoh K, Suzuki S. An error taxonomy system for analysis of haemodialysis inci-dents. J Ren Care. 2014;40(4):239-48.

31. Guerrero VM, García GP, Hidalgo CGO, Henández de Arribas V, Penã JR. Nivel de seguri-dad percibida por el paciente de hemodiálisis crónica. Enferm nefrol. 2016;19(3):255-263. 